\title{
Nível de atividade física e fatores de risco cardiometabólico em usuários da Atenção Básica à Saúde
}

http://dx.doi.org/10.11606/1807-5509202000020305

\author{
Carlos Alberto da SILVA* \\ Yuri Alberto FREIRE** \\ Francisco José Rosa de SOUZA* \\ Pabyle Alves FLAUZINO*** \\ Altieres Elias de Sousa JUNIOR** \\ Francisco Sérgio Lopes Vasconcelos FILHO ${ }^{* * * * *}$ \\ Eduardo Caldas COSTA**
}

*Instituto de Educação Física e Esportes, Universidade Federal do Ceará, Fortaleza, CE, Brasil.

${ }^{* *}$ Departamento de Educação Física, Universidade Federal do Rio Grande do Norte, Natal, RN, Brasil. ${ }^{* * *}$ Hospital Universitário Walter Cantídio, Universidade Federal do Ceará, Fortaleza,

$\mathrm{CE}$, Brasil.

${ }^{* * * *}$ Instituto Superior de Ciências Biomédicas, Universidade Estadual do Ceará, Fortaleza, CE, Brasil.

\section{Introdução}

No Brasil, as doenças crônico-degenerativas não transmissíveis, especialmente as de etiologia cardiometabólica, se configuram como o principal fator de morbidade e mortalidade, o que gera um custo substancial para os cofres públicos ${ }^{1}$. Em 2014, somente a hipertensão arterial sistêmica (HAS) e o diabetes mellitus (DM) foram responsáveis por 120 mil mortes ${ }^{2}$. Apesar da implementação de Políticas específicas para reduzir a carga destas doenças (e.g., HiperDia), nos últimos 10 anos, a prevalência de HAS e DM aumentaram em 14\% e $68 \%$, respectivamente ${ }^{3}$.
Dados da pesquisa VIGITEL ${ }^{3}$ (i.e., Vigilância de Fatores de Risco e Proteção para Doenças Crônicas por Inquérito Telefônico) mostram que a prevalência da HAS e DM na população adulta residente nas capitais brasileiras é de 25,7 e 8,9\%, respectivamente, sendo maior no sexo feminino e com o avançar da idade. Adicionalmente, o excesso de peso corporal (i.e., índice de massa corporal maior ou igual a $25 \mathrm{~kg} / \mathrm{m}^{2}$ ) é um importante fator de risco para a HAS e $\mathrm{DM}^{4}$. Na última década, a prevalência de brasileiros com excesso de peso aumentou em $26 \%$ e, atualmente, mais da metade 
da população apresenta esta condição ${ }^{3}$. Além disso, é importante destacar que a obesidade abdominal está diretamente associada à doença cardiometabólica, mesmo naqueles sem excesso de peso ${ }^{5}$.

A atividade física atua na prevenção e tratamento de diversas doenças cardiometabólicas ${ }^{6}$. Apesar dos benefícios da prática regular da atividade física, $45 \%$ dos brasileiros não cumprem a recomendação mínima de atividade física para a saúde (i.e., 150 minutos de atividade física moderada ou 75 minutos de vigorosa por semana), e este percentual é maior no sexo feminino e com o avançar da idade ${ }^{3}$. No Brasil, a inatividade física foi responsável por 8,2\% dos casos de doença arterial coronariana, 10,1\% dos casos de DM, bem como $13,2 \%$ da mortalidade por todas as causas, gerando uma redução de 1,08 anos na expectativa de vida da população ${ }^{7}$.

Segundo a Organização Mundial da Saúde ${ }^{8}$, a inatividade física, os níveis elevados de pressão arterial e glicemia, o excesso de peso e o fumo são as principais causas de morte no mundo. No Brasil, a prevalência destes fatores oscila entre os sexos e as faixas-etárias, no entanto, todos eles aumentam com a baixa condição socioeconômica ${ }^{3}$. A população brasileira com baixa condição socioeconômica, principalmente da região Nordeste, utiliza, majoritariamente, os serviços de saúde financiados pelo Sistema Único de Saúde ${ }^{9}$, onde a atenção básica de saúde é a porta de entrada para a utilização destes serviços em todos os níveis de complexidade ${ }^{10}$. A atenção básica de saúde é caracterizada por um conjunto de ações que envolvem a promoção e a proteção da saúde, a prevenção de agravos, o diagnóstico, o tratamento, a reabilitação e a manutenção da saúde ${ }^{11}$.

Portanto, considerando o impacto da inatividade física e dos indicadores de risco cardiometabólico sobre a saúde pública, especialmente na Atenção Básica à Saúde, ações de caráter diagnóstico, certamente, podem auxiliar no planejamento de açôes com objetivo de controlar os fatores de risco e, consequentemente, reduzir a morbidade e mortalidade precoce. Neste sentido, investigar o nível de atividade física e a prevalência de fatores de risco cardiometabólico entre usuários da atenção básica de saúde de forma estratificada (i.e., por sexo e faixa-etária) se faz necessário. Portanto, o objetivo deste estudo foi analisar o nível de atividade física e a prevalência de fatores de risco cardiometabólico em usuários da atenção básica de saúde.

\section{Método}

O presente estudo é de natureza transversal, realizado na cidade de Fortaleza-CE, cujos dados foram coletados no período compreendido entre setembro de 2014 à maio de 2015. O estudo foi realizado nas 91 Unidades Básicas de Saúde das seis regionais da cidade de Fortaleza-CE. Seguindo o parâmetro amostral estratificado por conveniência, foram avaliados 20 indivíduos de cada Unidade Básica de Saúde ( $\mathrm{n}=1820$ ).

Foram avaliados indivíduos de ambos os sexos com idade igual ou superior a 30 anos e sem doença funcionalmente incapacitante. O projeto foi aprovado pelo Comitê de Ética em Pesquisa do Hospital Universitário Walter Cantídio, Universidade Federal do Ceará, sob o parecer de no 1.360 .536 . Todos os voluntários assinaram um termo de Consentimento Livre e Esclarecido para participação no estudo, de acordo com a Resolução 466/2012 do Sistema Nacional de Saúde. A amostra foi categorizada de acordo com a faixa etária em: adultos $(30-39)$, meia-idade (40 - 59 anos) e idosos (acima de 60 anos). Além disso, as análises foram separadas também entre os sexos. Os seguintes aspectos foram considerados: nível de atividade física, participação em programa de exercício físico, excesso de peso, obesidade abdominal, tabagismo e diagnóstico de HAS e DM.

O nível de atividade física dos participantes foi avaliado utilizando-se a versão curta do International Physical Activity Questionnaire (IPAQ). Este instrumento avalia o tempo semanal de atividade física moderada e vigorosa em períodos maior ou igual a 10 minutos $^{12}$. Um escore de atividade física em minutos por semana foi calculado somandose os minutos despendidos em atividades de intensidade moderada por semana com os minutos despendidos em atividades de intensidade vigorosa multiplicado por dois ${ }^{13}$. Tal estratégia visa considerar as diferentes intensidades de cada atividade e está de acordo com as recomendaçôes atuais quanto à prática de atividade física ${ }^{14}$. Os indivíduos foram classificados como fisicamente ativos (i.e., escore $>150$ ), insuficientemente ativos (i.e., escore < 150) ou fisicamente inativos (i.e., escore $=0$ ). 
Adicionalmente, os participantes foram questionados sobre a realização de atividade física regular no lazer ou tempo livre. Foi considerada qualquer atividade física realizada de forma frequente, como um programa de exercício físico estruturado ou caminhada não supervisionada.

A massa corporal foi avaliada por uma balança tipo plataforma (Líder ${ }^{\circledR}$, modelo LD1050, São Paulo, Brasil); a estatura, por meio de um estadiômetro acoplado a própria balança, com capacidade para 2 $\mathrm{m}$ e precisão de $1 \mathrm{~mm}$ e; circunferência da cintura, mensurada por meio de fita métrica (Seca ${ }^{\circledR}$, modelo 201, São Paulo, Brasil), inelástica e flexível com escala de $0-205 \mathrm{~cm}$. As avaliaçôes antropométricas foram realizadas conforme MATSUDO ${ }^{15}$.

O excesso de peso foi avaliado pelo índice de massa corporal (IMC) e a obesidade abdominal, pela relação cintura/estatura, que são indicadores antropométricos de risco cardiometabólico ${ }^{16}$. Para o cálculo do IMC foi utilizado a Fórmula de Quetelet, em que IMC é igual à massa corporal $(\mathrm{kg})$ dividido pelo quadrado da altura (m). Classificou-se com excesso de peso o adulto e idoso que apresentou IMC maior que 25 $\mathrm{kg} / \mathrm{m}^{2}$ e $27 \mathrm{~kg} / \mathrm{m}^{2}$ respectivamente ${ }^{17,18}$. O cálculo da relação cintura/estatura foi realizado pela divisão da circunferência da cintura $(\mathrm{cm})$ pela medida da estatura $(\mathrm{cm})$, em que os pontos de corte utilizados para a classificação de risco cardiometabólico para homens e mulheres foram acima de 0,52 e 0,53, respectivamente ${ }^{5}$. O uso de tabaco foi questionado com a seguinte pergunta: "Atualmente você fuma?". A presença de HAS e DM2 foi avaliada de forma auto-reportada por meio da seguinte pergunta: "Algum médico ou profissional da saúde já lhe disse que você era hipertenso ou diabético?".

As variáveis quantitativas foram apresentadas como média \pm desvio-padrão, enquanto as qualitativas foram apresentadas por distribuição de frequência absoluta e relativa. Para a estatística inferencial, foi aplicado o teste qui-quadrado para comparação de proporçōes (i.e., variáveis dependentes entre grupos etários e sexo) e o teste z com ajuste de Bonferroni para comparação entre colunas (i.e., entre grupos etários e sexo). Para a análise dos dados foi utilizado o software SPSS Statistics 22.0, adotando como nível de significância estatística o valor de $\mathrm{p} \leq 0,05$.

\section{Resultados}

Dos 1820 indivíduos entrevistados, 293 estavam com os dados incompletos e foram excluídos da análise. Portanto, 1527 foram incluídos na análise (i.e., $83,9 \%$ da amostra calculada inicialmente). A idade média da amostra foi de 52,7 $\pm 13,0$ anos, sendo representada por $1.109(72,6 \%)$ mulheres e $418(27,4 \%)$ homens, dos quais $279(18,3 \%)$ foram adultos, 785 (51,4\%) meia-idade e 463 (30,3\%) idosos. Não houve diferença na idade média entre homens $(52,6 \pm 13,3$ anos) e mulheres $(52,7 \pm$ 12,9 anos).

A TABELA 1 apresenta o nível de atividade física, avaliado pelo IPAQ, e fatores de risco cardiometabólico entre homens e mulheres.
A prevalência de indivíduos que atenderam as recomendações de atividade física (i.e., classificados como fisicamente ativos) foi maior entre as mulheres $(63,0 \%$ vs. $36,8 \%, \mathrm{p}<0,05)$. Além disso, as mulheres relataram maior participação em atividade física no lazer $(\mathrm{p}<0,05)$. Identificou-se também uma alta prevalência de homens que não são fisicamente ativos (i.e., 63,1\%). Não houve diferença significativa entre os sexos em relação ao IMC, risco cardiometabólico pela relação cintura/ estatura, bem como na prevalência de HAS e DM ( $\mathrm{p}>0,05)$. Quanto ao uso de tabaco, os homens apresentaram maior prevalência (i.e., $11,7 \%$ vs. $7,3 \%, \mathrm{p}<0,05)$.

TABELA 1 -Nível de atividade física e fatores de risco cardiometabólico entre homens e mulheres usuários da Atenção Básica à Saúde de Fortaleza - CE.

\begin{tabular}{lcccc}
\hline & Total $\mathbf{n}(\mathbf{\%})$ & Homens $\mathbf{n}(\mathbf{\%})$ & Mulheres n (\%) & p \\
\hline Nível de Atividade Física & & & & \\
Inativo & $511(33,5)$ & $207(49,5)$ & $304(27,4)^{*}$ & \\
Insuficientemente Ativo & $163(10,6)$ & $57(13,7)$ & $106(9,6)^{*}$ & $<0,001$ \\
Fisicamente Ativo & $853(55,9)$ & $154(36,8)$ & $699(63,0) *$ & \\
\hline
\end{tabular}

continua 
continuação

\begin{tabular}{|c|c|c|c|c|}
\hline & Total n (\%) & Homens n (\%) & Mulheres n (\%) & $\mathrm{p}$ \\
\hline \multicolumn{5}{|c|}{ Atividade Física de Lazer } \\
\hline Não & $1361(89,1)$ & $401(95,9)$ & $960(86,6)^{*}$ & \multirow{2}{*}{$<0,001$} \\
\hline Sim & $166(10,9)$ & $17(4,1)$ & $149(13,4)^{*}$ & \\
\hline \multicolumn{5}{|c|}{ Índice de massa corporal } \\
\hline Peso normal & $625(40,9)$ & $156(37,3)$ & $469(42,3)$ & \multirow{2}{*}{0,078} \\
\hline Excesso de peso & $902(59,1)$ & $262(62,7)$ & $640(57,7)$ & \\
\hline \multicolumn{5}{|c|}{ Relação cintura/altura } \\
\hline Sem risco & $527(34,5)$ & $128(30,6)$ & $399(36,0)$ & \multirow{2}{*}{0,053} \\
\hline Com Risco & $1000(65,5)$ & $290(69,4)$ & $710(64,0)$ & \\
\hline \multicolumn{5}{|l|}{ Tabagismo } \\
\hline Não & $1397(91,5)$ & $369(88,3)$ & $1028(92,7)^{*}$ & \multirow{2}{*}{0,007} \\
\hline Sim & $130(8,5)$ & $49(11,7)$ & $81(7,3)^{*}$ & \\
\hline \multicolumn{5}{|c|}{ Hipertensão Arterial Sistêmica } \\
\hline Não & $1065(69,7)$ & $298(71,3)$ & $767(69,2)$ & \multirow{2}{*}{0,454} \\
\hline $\operatorname{Sim}$ & $462(30,3)$ & $120(28,7)$ & $342(30,8)$ & \\
\hline \multicolumn{5}{|c|}{ Diabetes mellitus } \\
\hline Não & $1345(88,1)$ & $370(88,5)$ & $975(87,9)$ & \multirow{2}{*}{0,411} \\
\hline Sim & $182(11,9)$ & $48(11,5)$ & $134(12,1)$ & \\
\hline
\end{tabular}

Peso normal $=\mathrm{IMC}<25 \mathrm{~kg} / \mathrm{m}^{2}$ e $27 \mathrm{~kg} / \mathrm{m}^{2}$ para adultos e idosos, respectivamente; excesso de peso $=\mathrm{IMC} \geq 25 \mathrm{~kg} / \mathrm{m}^{2}$ e $27 \mathrm{~kg} / \mathrm{m}^{2}$ para adultos e idosos, respectivamente; sem risco = relação cintura/estatura $\leq 0,52$ e 0,53 para homens e mulheres, respectivamente; com risco $=$ relação cintura/estatura $>0,52$ e 0,53 para homens e mulheres, respectivamente. ${ }^{*}=$ diferença significativa $(\mathrm{p} \leq 0,05)$ com os homens de acordo com teste $\mathrm{z}$.

A TABELA 2 apresenta o nível de atividade física e a prevalência de fatores de risco cardiometabólico da população adulta, de meia-idade e idosa. A prevalência de sujeitos fisicamente ativos foi menor em idosos $(\mathrm{p}<0,05)$, apesar dos mesmos apresentarem maior participação em atividade física no lazer $(\mathrm{p}<0,05)$ quando comparado aos adultos. Com relação ao IMC, 59,1\% da amostra apresentaram excesso de peso, com maior prevalência na população adulta e de meia-idade $(\mathrm{p}<0,05)$. No tocante ao risco cardiometabólico pela relação cintura/estatura, os idosos apresentaram maior prevalência $(\mathrm{p}<0,05)$, apesar de que em todas as faixas-etárias, mais da metade evidenciaram risco alto. Em relação ao tabagismo, $8,5 \%$ da amostra são fumantes, sem diferença entre as faixas-etárias ( $p>0,05)$.

TABELA 2 -Nível de atividade física e fatores de risco cardiometabólico da população adulta, de meia-idade e idosa usuária da Atenção Básica à Saúde de Fortaleza - CE.

\begin{tabular}{|c|c|c|c|c|}
\hline & Adultos n (\%) & Meia-idade n (\%) & Idosos n (\%) & $\mathrm{p}$ \\
\hline \multicolumn{5}{|l|}{ Nível de Atividade Física } \\
\hline Inativo & $69(24,7)$ & $252(32,1)$ & $190(41,0)^{* t}$ & \multirow{3}{*}{$<0,001$} \\
\hline Insuficientemente Ativo & $32(11,5)$ & $86(11,0)$ & $45(9,8)$ & \\
\hline Fisicamente Ativo & $178(63,8)$ & $447(56,9)$ & $228(49,2)^{*} \boldsymbol{t}$ & \\
\hline \multicolumn{5}{|l|}{ Atividade Física de Lazer } \\
\hline Não & $259(92,8)$ & $711(90,6)$ & $391(84,4)^{*}$ & \multirow{2}{*}{$<0,001$} \\
\hline $\operatorname{Sim}$ & $20(7,2)$ & $74(9,4)$ & $72(15,6) * \dagger$ & \\
\hline \multicolumn{5}{|c|}{ Índice de massa corporal } \\
\hline Peso normal & $84(30,1)$ & $277(35,3)$ & $264(57,9)^{* t}$ & \multirow{2}{*}{$<0,001$} \\
\hline Excesso de peso & $195(69,9)$ & $508(64,7)$ & $199(43,1)^{*}+$ & \\
\hline
\end{tabular}


continuação

\begin{tabular}{|c|c|c|c|c|}
\hline & Adultos n (\%) & Meia-idade n (\%) & Idosos n (\%) & $\mathrm{p}$ \\
\hline \multicolumn{5}{|c|}{ Relação cintura/altura } \\
\hline Sem risco & $115(41,2)$ & $266(33,9)$ & $146(31,5)^{*}$ & \multirow{2}{*}{0,023} \\
\hline Com Risco & $164(58,8)$ & $519(66,1)$ & $317(68,5)^{*}$ & \\
\hline \multicolumn{5}{|l|}{ Tabagismo } \\
\hline Não & $258(92,5)$ & $713(90,8)$ & $426(92,0)$ & \multirow{2}{*}{0,632} \\
\hline $\operatorname{Sim}$ & $21(7,5)$ & $72(9,2)$ & $37(8,0)$ & \\
\hline \multicolumn{5}{|c|}{ Hipertensão Arterial Sistêmica } \\
\hline Não & $247(88,5)$ & $556(70,8)^{*}$ & $262(56,6)^{*} t$ & \multirow{2}{*}{$<0,001$} \\
\hline $\operatorname{Sim}$ & $32(11,5)$ & $229(29,2)^{*}$ & $201(43,4) * t$ & \\
\hline \multicolumn{5}{|c|}{ Diabetes mellitus } \\
\hline Não & $271(97,1)$ & $703(89,6)^{*}$ & $371(80,1)^{*}+$ & \multirow{2}{*}{$<0,001$} \\
\hline Sim & $8(2,9)$ & $82(10,4)^{*}$ & $92(19,9)^{*}+$ & \\
\hline
\end{tabular}

Peso normal $=\mathrm{IMC}<25 \mathrm{~kg} / \mathrm{m}^{2}$ e $27 \mathrm{~kg} / \mathrm{m}^{2}$ para adultos e idosos, respectivamente; excesso de peso $=\mathrm{IMC} \geq 25 \mathrm{~kg} / \mathrm{m}^{2}$ e $27 \mathrm{~kg} / \mathrm{m}^{2}$ para adultos e idosos, respectivamente; sem risco = relação cintura/estatura $\leq 0,52$ e 0,53 para homens e mulheres, respectivamente; com risco = relação cintura/estatura $>0,52$ e 0,53 para homens e mulheres, respectivamente. ${ }^{*}=$ diferença significativa $(\mathrm{p} \leq 0,05)$ com adultos de acordo com teste $\mathrm{z} . \mathbf{t}=$ diferença significativa $(\mathrm{p} \leq 0,05)$ com indivíduos de meia-idade de acordo com teste $\mathrm{z}$.

\section{Discussão}

O objetivo deste estudo foi analisar o nível de atividade física e a prevalência de fatores de risco cardiometabólico em usuários da Atenção Básica à Saúde. Os principais achados desse estudo foram: i) há alta prevalência de usuários da Atenção Básica à Saúde que não atingem as recomendaçóes de atividade física para saúde, em especial os homens (i.e., 63,1\%) e idosos (i.e., 50,7\%); ii) cerca de $90 \%$ da população investigada não realiza nenhuma atividade física regular no lazer; iii) a população apresenta alta prevalência de excesso de peso (i.e., 59,1\%) e obesidade abdominal (i.e., 65,5\%); iv) a prevalência de HAS (i.e., -30\%) e DM (i.e., -12\%) foi similar entre os sexos e maior com a avançar da idade (em idosos, HAS - 45\% e DM - 20\%).

A amostra foi constituída por apenas $27,4 \%$ de homens, o que destaca a baixa procura desta população aos serviços de saúde, muito devido a suas características comportamentais como pressa, objetividade, medo e resistência ${ }^{19}$. Ainda, entre a população masculina, metade não realiza nenhuma atividade física moderada a vigorosa por semana, $96 \%$ não participa de atividade física regular no lazer e $12 \%$ são tabagistas. As mulheres apresentaram menores prevalências em todos os aspectos mencionados acima, entretanto, houve semelhança nos demais indicadores de risco, o que sugere que as mulheres usuárias da atenção básica de saúde sofrem por outros fatores não investigados neste estudo, onde se dá a importância de políticas específicas para os sexos, tal como a Política Nacional de Atenção Integral à Saúde do $\mathrm{Homem}^{20}$, que visa estimular, principalmente, o autocuidado e a facilitação à assistência a saúde dos homens, bem como a Política Nacional de Atenção Integral à Saúde da Mulher, que busca a humanização da atenção à saúde, com ações específicas para as diferentes fases da vida da mulher ${ }^{21}$.

Em relação ao uso do tabaco, $8,5 \%$ da amostra fazia uso. Apesar desta prevalência ser menor em relação à nacional $(10,8 \%)^{3}$, é de grande importância políticas de saúde que visem conscientizar a cessação do uso de tabaco, uma vez que é um fator relevante à morbimortalidade e custeios de doenças cardiovasculares, pulmonares e diversos tipos de câncer, totalizando um custo de 23,37 bilhões de reais em $2011^{22}$.

Foi identificado maior prevalência de inatividade física em idosos, apesar de esta população apresentar maior participação em atividade física regular no lazer. Certamente, a redução da atividade física habitual que acompanha o envelhecimento seja um dos principais fatores para os baixos níveis de atividade física nos idosos ${ }^{23}$. Além do mais, mesmo 
nos idosos, a participação em atividade física no lazer é baixa (i.e., 15,6\%), o que põe em prova a eficácia dos programas existentes que visam o aumento dos níveis de atividade física da população na Atenção Básica à Saúde. O aconselhamento à prática de atividade física é uma estratégia custoeficiente que deve ser utilizado na Atenção Básica à Saúde ${ }^{24,25}$. Em uma revisão sobre aconselhamento à prática de atividade física baseado no modelo dos cinco A's (avaliar [assess], aconselhar [advise], concordar [agree], assistir [assist] e arranjar/organizar [arrange] $)^{24}$, foi demonstrado que os sujeitos que participaram das intervençôes de intensidade moderada (i.e., 31 - 360 minutos) aumentaram 33 minutos de atividade física por semana e tinham $22 \%$ a mais de chance de atender as diretrizes de atividade física após seis e 12 meses. Isso implica em benefícios de grande magnitude para a saúde pública, tendo em vista que uma redução de $25 \%$ na inatividade física mundial reduziria mais de 1,3 milhōes de mortes a cada ano ${ }^{7}$.

Foi encontrada relação direta entra as doenças cardiometabólicas e o avançar da faixa-etária. Adicionalmente, identificou-se alta prevalência de HAS e DM já em indivíduos de meia-idade, em que suas proporções triplicaram, quando comparado aos adultos. Quanto ao excesso de peso, identificou-se uma alta prevalência de indivíduos nesta condição, principalmente em adultos (i.e., 69,9\%) e meiaidade (i.e., 64,7\%). Segundo a Organização Mundial de Saúde ${ }^{8}$, o excesso de peso é um dos cinco principais fatores de risco para mortalidade mundial. Além disso, $65,5 \%$ da amostra estudada estão em risco cardiometabólico pela presença de obesidade abdominal, que indica risco cardiometabólico aumentado mesmo em indivíduos eutróficos ${ }^{5,16}$. Portanto, abordagens de prevenção e promoção da saúde devem ser reforçadas ainda na idade adulta, visando o controle de peso e do perfil cardiometabólico da população, especialmente direcionadas ao aumento dos níveis de atividade física e à reeducação alimentar ${ }^{26}$.

Apesar dos achados interessantes do presente estudo, algumas limitaçóes devem ser mencionadas. Primeiro, o nível de atividade física e a presença de doenças cardiometabólicas (i.e., HAS e DM) foram avaliados de forma auto-reportada, o que pode ter subestimado ou superestimado o nível de atividade física e a prevalência dessas doenças na nossa amostra. É importante destacar que, de fato, informações mais objetivas sobre o nível de atividade física nos diferentes domínios (i.e., lazer, transporte/ deslocamento, atividades domésticas e laborais/ ocupacionais) são necessárias para aprofundar o entendimento sobre tal comportamento em usuários da Atenção Básica à Saúde. Segundo, a amostra foi selecionada por conveniência, o que pode ter gerado viés de seleção dos participantes. Portanto, nossos resultados devem ser interpretados com cautela. Além disso, diante da dimensão continental do Brasil, é importante destacar que os achados do nosso estudo, realizado na cidade de Fortaleza-CE, não necessariamente refletem a realidade de outros estados e regiōes.

Em conclusão, foi identificada uma alta prevalência de usuários da atenção básica de saúde que não atingem as recomendações mínimas de atividade física para saúde, principalmente homens e idosos. Além disso, a grande maioria não participa de nenhuma atividade física regular no lazer. A prevalência dos fatores de risco cardiometabólico foi maior em indivíduos de meia-idade e idosos; entretanto, o excesso de peso foi um fator de risco com destacada prevalência, independente da faixa etária e sexo. Portanto, mesmo com os avanços da atenção básica de saúde para garantir proximidade à vida da população, vínculo e atividades que busquem a promoção da saúde, o presente trabalho mostra que ainda são necessários esforços para o aumento dos níveis de atividade física e redução dos fatores de risco cardiometabólico em usuários da atenção básica em saúde.

\section{Abstract}

Physical activity level and cardiometabolic risk factors in primary healthcare system users

The aim of this study was to analyze the physical activity levels and the prevalence of cardiometabolic risk factors in the primary healthcare system users. A total of 1527 individuals $(52,7 \pm 13,0$ years; $72,6 \%$ women) aged over 30 years from Fortaleza-CE participated of this study. Physical activity level, leisure time physical activity, body weight excess, abdominal obesity, smoking, hypertension and diabetes mellitus were analyzed. The independent variables were sex and age group (adult, middle-aged and elderly). Our results 
showed that men presented a lower physical activity level $(p<0,05)$, lower engagement in leisure time physical activity $(p<0,05)$ and higher smoking levels $(p<0,05)$. The elderly showed lower physical activity levels $(p<0,05)$ than adults and middle-aged individuals, even showing higher participation in leisure time physical activity $(p<0,05)$. There was a high prevalence of body weight excess $(59.1 \%)$ and abdominal obesity (65.5\%). The prevalence of body weight excess was higher in adults (69.9\%) and middle-aged (64.7\%) individuals. Regarding the prevalence of hypertension and diabetes mellitus, there was a significant increase from the middle-age range, without differences between sexes. In conclusion, we found a high prevalence of physical inactivity, mainly in men and in the elderly, as well as a higher prevalence of cardiometabolic risk factors in middle-aged and elderly individuals.

KeYwords: Unified Health System; Cardiovascular Diseases; Systemic Arterial Hypertension; Diabetes Mellitus; Exercise.

\section{Referências}

1. Abegunde DO, Mathers CD, Adam T, Ortegon M, Strong K. The burden and costs of chronic diseases in low-income and middle-income countries. Lancet. 2007;370(9603):1929-38.

2. Brasil. Banco de dados do Sistema Único de Saúde - DATASUS. Informações de Saúde, Sistema de Informações sobre Mortalidade. Brasília: Ministério da Saúde [citado 12 fev 2018]. Disponível em: <http://tabnet.datasus.gov.br/cgi/ deftohtm.exe?sim/cnv/obt10uf.def>.

3. Vigitel Brasil 2016: Vigilância de fatores de risco e proteção para doenças crônicas por inquérito telefônico: estimativas sobre frequência e distribuição sociodemográfica de fatores de risco e proteçáo para doenças crônicas nas capitais dos 26 estados brasileiros e no Distrito Federal em 2016 / Ministério da Saúde, Secretaria de Vigilância em Saúde, Departamento de Vigilância de Doenças e Agravos não Transmissíveis e Promoção da Saúde. - Brasília: Ministério da Saúde; 2017.

4. Mokdad AH, Ford ES, Bowman BA, Dietz WH, Vinicor F, Bales VS, at al. Prevalence of obesity, diabetes, and obesityrelated health risk factors, 2001. Jama. 2003;289(1):76-79.

5. Haun DR, Pitanga FJG, Lessa I. Razão cintura/estatura comparado a outros indicadores antropométricos de obesidade como preditor de risco coronariano elevado. Rev Assoc Med Bras. 2009; 55(6): 705-711.

6. Booth FW, Roberts CK, Laye MJ. Lack of exercise is a major cause of chronic diseases. Compr Physiol. 2012;2(2):11431211

7. Lee MI, Shiroma EJ, Lobelo F, Puska P, Blair SN, Katzmarzyk PT. Effect of physical inactivity on major noncommunicable diseases worldwide: an analysis of burden of disease and life expectancy. Lancet. 2012;380:219-29.

8. World Health Organization. Global health risks: mortality and burden of disease attributable to selected major risks. Genebra: World Health Organization; 2009.

9. Porto SM, Ugá MAD, Moreira RS. Uma análise da utilização de serviços de saúde por sistema de financiamento: Brasil 1998-2008. Cien Saude Colet. 2011;16(9):3795-3806.

10. Marsiglia RMG. Universalização do acesso ao Sistema Único de Saúde no Brasil: desafios para a Atenção Primária à Saúde. Cad Ter Ocup UFSCar. 2012;20(3):317-325.

11. Brasil. Ministério da Saúde. Aprova a Política Nacional de Atenção Básica, estabelecendo a revisão de diretrizes e normas para a organizaçáo da Atenção Básica para o Programa Saúde da Família (PSF) e o Programa Agentes Comunitários de Saúde (PACS). Portaria n. 648/GM, 28 março 2006. Diário Oficial da União, Brasília (2006 mar. 29); Sec.1:71-6.

12. Guedes DP, Lopes CC, Guedes JERP. Reprodutibilidade e validade do Questionário Internacional de Atividade Física em adolescentes. Rev Bras Med Esporte. 2005;11(2):151-8.

13. Hallal PC, Tenório MCM, Tassitano RM, Reis RS, Carvalho YM, Cruz DKA, et al. Avaliação do programa de promoçáo da atividade física Academia da Cidade de Recife, Pernambuco, Brasil: percepçóes de usuários e náo-usuários. Cad Saude Publica. 2010;26(1):70-8.

14. Garber CE, Blissmer B, Deschenes MR, Franklin BA, Lamonte MJ, Lee IM, et al. American College of Sports Medicine position stand: Quantity and quality of exercise for developing and maintaining cardiorespiratory, musculoskeletal, and neuromotor fitness in apparently healthy adults: guidance for prescribing exercise. Jour Med and Science in Sports 
and Exercise. 2011;43(7):1334-1359.

15. Matsudo SMM. Avaliação do Idoso: física e funcional. Londrina: Midiograf; 2000.

16. Zhu Q, Shen FYT, Zhou Q, Deng H, Gu X. Waist-to-height ratio is an appropriate index for identifying cardiometabolic risk in Chinese individuals with normal body mass index and waist. Jour of diabetes. 2014;6(6):527-534.

17. World Health Organization. Diet, Nutrition and prevention of chronic disease. Report of a WHO study group. Technical Report Series 797. Geneva: WHO; 1990.

18. Lipschitz DA. Screening for nutritional status in the elderly. Prim Care. 1994;21:55-67.

19. Knauth DR, Couto MT, Figueiredo WDS. A visão dos profissionais sobre a presença e as demandas dos homens nos serviços de saúde: perspectivas para a análise da implantação da Política Nacional de Atenção Integral à Saúde do Homem. Cien Saude Colet. 2012;17(10):2617-2626.

20. Brasil. Secretaria de Atenção à Saúde. Política nacional de atenção integral à saúde do homem. Brasília: Ministério da Saúde; 2008.

21. Brasil. Secretaria de Atenção à Saúde. Departamento de Ações Programáticas Estratégicas. Política nacional de atenção integral à saúde da mulher: princípios e diretrizes / Ministério da Saúde, Secretaria de Atenção à Saúde, Departamento de Ações Programáticas Estratégicas. Brasília: Ministério da Saúde; 2004.

22. Pinto MT, Pichon-Riviere A, Bardach A. The burden of smoking-related diseases in Brazil: mortality, morbidity and costs. Cad saude publica. 2015;31(6):1283-1297.

23. Tassitano R, Mielke G, Carvalho W, Oliveira M, Malta D. Tendência temporal de indicadores da prática de atividade física e comportamento sedentário nas capitais da Região Nordeste do Brasil: 2006-2013. Rev Bras Ativi Física Saúde. 2015;20(2):152-164.

24. Lin JS, O'Connor E, Whitlock EP, Beil TL. Behavioral counseling to promote physical activity and a healthful diet to prevent cardiovascular disease in adults: a systematic review for the US Preventive Services Task Force. Ann int med. 2010;153(11):736-750.

25. Goldstein MG, Whitlock EP, DePue J. Multiple behavioral risk factor interventions in primary care: summary of research evidence. Amer jour of prev medicine. 2004;27(2):61-79.

26. Seligman BG, Polanczyk CA, Santos AS, Foppa M, Junges M, Bonzanini L, et al. Intensive practical lifestyle intervention improves endothelial function in metabolic syndrome independent of weight loss: a randomized controlled trial. Rev Metabolism. 2011;60(12):1736-1740.

ENDEREÇO DE CORRESPONDÊNCIA: Carlos Alberto da Silva Campus do Pici, Av. Mister Hull, s/n Fortaleza - CE - BRASIL CEP: 60455-760

E-mail: carlosas@gmail.com; carlosas@ufc.br
Submetido: $14 / 11 / 2017$

Revidado: 15/02/2018

Aceito: 09/04/2018 